\title{
DESTRUCTION OF LAGRANGIAN TORUS FOR POSITIVE DEFINITE HAMILTONIAN SYSTEMS
}

\author{
Chong-Qing Cheng \& Lin Wang
}

\begin{abstract}
For an integrable Hamiltonian $H_{0}=\frac{1}{2} \sum_{i=1}^{d} y_{i}^{2}(d \geq 2)$, we show that any Lagrangian torus with a given unique rotation vector can be destructed by arbitrarily $C^{2 d-\delta}$-small perturbations. In contrast with it, it has been shown that KAM torus with constant type frequency persists under $C^{2 d+\delta}$-small perturbations $([\mathrm{P}])$.
\end{abstract}

Key words. Lagrangian torus, nearly integrable Hamiltonian system, action minimizing orbit

AMS subject classifications (2000). 37J40, 37J50, 70H08, 58H27

\section{INTRODUCTION AND MAIN RESULT}

For exact and area-preserving twist maps on annulus, it was proved by Herman in [H1] that invariant circles can be destructed by $C^{3-\delta}$ arbitrarily small perturbations where $\delta$ is a small positive constant. A variational proof of a similar result was provided in [W]. For certain rotation numbers, it was obtained by Mather (resp. Forni) in [Ma1] (resp. [F]) that the invariant circles with those rotation numbers can be destroyed by small perturbations in finer topology respectively. More precisely, Mather considered Liouville rotation numbers and the topology of the perturbation induced by $C^{\infty}$ metric. Forni was concerned about more special rotation numbers which can be approximated by rational ones exponentially and the topology of the perturbation induced by the supremum norm of $C^{\omega}$ (real-analytic) function. For certain positive definite systems with $d$-degrees of freedom, Herman found that all invariant Lagrangian tori can be destructed by $C^{d+1-\delta}$ arbitrarily small perturbation in [H3], where Lagrangian torus is a natural analogy to invariant circle in multi-degrees of freedom (see Definition 1.3 below).

In contrast with it, the KAM theory claims the existence of $d$-dimensional invariant tori in nearly integrable systems with $d$ degrees of freedom. More precisely, Moser proved that the invariant circle with constant type frequency of an integrable area-preserving twist map is persisted under arbitrarily small perturbations in the $C^{333}$ topology ([Mo1]). Due to the efforts of Moser, Rüssman, Herman and Pöschel ( $\mathrm{H1}, \mathrm{H} 2$, Mo2, Mo3, P, R1, R2), it was obtained that certain invariant tori are persisted under arbitrarily small perturbations in the $C^{2 d+\delta}$ topology for Hamiltonian systems with $d$-degrees of freedom under non-degeneracy conditions. In particular, Herman proved in [H2] that for area-preserving twist maps on annulus, certain invariant circles can be persisted under arbitrarily small perturbations in the $C^{3}$ topology.

Comparing the results on both directions, it is natural to ask whether the $C^{2 d+\delta}$ condition can be reduced to $C^{r}$ condition $(r \leq 2 d)$ to ensure the existence 
of Lagrangian torus. In [C], it was proved that KAM torus with a given rotation vector does not exist if one carefully construct perturbations arbitrarily small in $C^{2 d-\delta}$ topology. But this does not imply non-existence of invariant Lagrangian torus. Indeed, it exists in the example in [C].

In this paper we prove the following:

Theorem 1.1 Given an integrable Hamiltonian $H_{0}(y)=\frac{1}{2} \sum_{i=1}^{d} y_{i}^{2}(d \geq 2)$, a rotation vector $\omega$ and a small positive constant $\delta$, there exists a sequence of $C^{\infty}$ Hamiltonians $\left\{H_{n}(x, y)\right\}_{n \in \mathbb{N}}$ such that $H_{n}(x, y) \rightarrow H_{0}(y)$ uniformly in $C^{2 d-\delta}$ topology and the Hamiltonian flow generated by $H_{n}(x, y)$ does not admit any Lagrangian torus with the rotation vector $\omega$, where $(x, y) \in \mathbb{T}^{d} \times \mathbb{R}^{d}$ and $\omega \in \mathbb{R}^{d}$.

This theorem implies that the rigidity of the Lagrangian torus is as the same as for the KAM torus. Roughly speaking, if no Lagrangian torus with the rotation vector $\omega$ survives under an arbitrarily small perturbation in $C^{r}$ topology, the maximum of $r$ is closely related to the arithmetic property of the rotation vector $\omega$. If $\omega$ is a constant type vector, then $r$ is at most $2 d-\delta$. If $\omega$ is a Liouville vector, then $r$ can be arbitrarily large. If $\omega$ can be approximated exponentially by rational vectors, then no Lagrangian torus with the rotation vector $\omega$ survives under an arbitrarily small perturbation in $C^{\omega}$ topology (see $[\mathrm{Be}]$ ).

In $\mathrm{T}^{*} \mathbb{T}^{d}$, a submanifold $\mathcal{T}^{d}$ is called a Lagrangian torus if it is diffeomorphic to the torus $\mathbb{T}^{d}$ and the non-degenerate closed 2-form vanishes on it. For positive definite Hamiltonian systems, if a Lagrangian torus is invariant under the Hamiltonian flow, it is then a graph over $\mathbb{T}^{d}$ (see $[\mathrm{BP}]$ ). An example of Lagrangian torus is any KAM torus.

Definition $1.2 \overline{\mathcal{T}}^{d}$ is called a d-dimensional KAM torus if

- $\overline{\mathcal{T}}^{d}$ is a Lipschitz graph over $\mathbb{T}^{d}$;

- $\overline{\mathcal{T}}^{d}$ is invariant under the Hamiltonian flow $\Phi_{t}^{H}$ generated by the Hamiltonian function $H$;

- there exists a diffeomorphism $\phi: \mathbb{T}^{d} \rightarrow \overline{\mathcal{T}}^{d}$ such that $\phi^{-1} \circ \Phi_{H}^{t} \circ \phi=R_{\omega}^{t}$ for any $t \in \mathbb{R}$, where $R_{\omega}^{t}: x \rightarrow x+\omega t$ and $\omega$ is called the rotation vector of $\overline{\mathcal{T}}^{d}$.

Generally, the rotation vector of the Lagrangian torus is not well defined if the Lagrangian torus contains several invariant sets with different rotation vectors. In this paper, we are only concerned with Lagrangian tori with given unique rotation vectors.

Definition $1.3 \mathcal{T}^{d}$ is called a d-dimensional Lagrangian torus with the rotation vector $\omega$ if

- $\mathcal{T}^{d}$ is a Lagrangian submanifold;

- $\mathcal{T}^{d}$ is invariant for the Hamiltonian flow $\Phi_{H}^{t}$ generated by $H$.

- each orbit on $\mathcal{T}^{d}$ has the same rotation vector.

In order to use a variational method, we recall the definition of action minimizing orbit for a positive definite Lagrangian system $L(x, v)$ satisfying superlinear growth with respect to $|v|_{x}$ (see [?]). 
Definition 1.4 An orbit $\gamma \in C^{a c}\left(\left[t_{1}, t_{2}\right], \mathbb{T}^{d}\right)$ (absolutely continuous) is called an action minimizing curve for $L$ on $\left[t_{1}, t_{2}\right]$ if

$$
\int_{t_{1}}^{t_{2}} L(\gamma(t), \dot{\gamma}(t)) d t \leq \int_{t_{1}}^{t_{2}} L(\bar{\gamma}(t), \dot{\bar{\gamma}}(t)) d t
$$

for every $\bar{\gamma} \in C^{a c}\left(\left[t_{1}, t_{2}\right], \mathbb{T}^{d}\right)$ satisfying

- $\gamma\left(t_{1}\right)=\bar{\gamma}\left(t_{1}\right), \gamma\left(t_{2}\right)=\bar{\gamma}\left(t_{2}\right)$;

- $\gamma$ and $\bar{\gamma}$ are in the same homotopy class, equivalently, their lifts to $\mathbb{R}^{d}$ connect the same points.

In [H4, it is proved that each orbit on $\mathcal{T}^{d}$ is an action minimizing curve. Actually, in order to prove Theorem 1.1, we only need the following properties of Lagrangian torus:

- it is a Lipschitz graph;

- every point possesses an action minimizing orbit with rotation vector $\omega$.

A rotation vector $\omega \in \mathbb{R}^{d}$ is called resonant if there exists $k \in \mathbb{Z}^{d}$ such that $\langle\omega, k\rangle=0$. Otherwise, it is non-resonant. The following approximation of the rotation vector is found in $\left[\mathrm{C}\right.$. For any given vector $\omega \in \mathbb{R}^{d}$ with $d \geq 2$, there is a sequence of integer vectors $k_{n} \in \mathbb{Z}^{d}$ with $\left|k_{n}\right| \rightarrow \infty$ as $n \rightarrow \infty$ such that

$$
\left|\left\langle\omega, k_{n}\right\rangle\right|<\frac{C}{\left|k_{n}\right|^{d-1}},
$$

where $C$ is a constant independent of $n$ and $|\cdot|$ denotes Euclidean norm, i.e. $|k|=$ $\left(\sum_{j=1}^{d} k_{i}^{2}\right)^{1 / 2}$ for $k=\left(k_{1}, k_{2}, \ldots, k_{d}\right)$. (we fix the norm once and for all.) If $\omega$ is nonresonant, this sequence contains infinitely many indivisible integer vectors. Here, we call the integer vector $k \in \mathbb{Z}^{d}$ indivisible integer vector if $\lambda k \notin \mathbb{Z}^{d}$ whenever $|\lambda|<1$.

Since a generic hyperbolic perturbation results in the transversality between the stable manifold and the unstable manifold, a Lagrangian torus with resonant rotation vector can be destructed by analytic perturbation arbitrarily close to zero. Hence, it is sufficient to consider a Lagrangian torus with non-resonant rotation vector.

The system we consider consists of one pendulum, a rotator with $d-1$ degrees of freedom and a perturbation coupling of them. By the transforation of coordinates (see (3.2)), the system with a given rotation vector $\omega$ can be reduced to the case with the rotation vector having a small first coordinate and a suitable large second coordinate. With variational methods, the full action of the system and the action of the pendulum near the separatrix are estimated. Moreover, it is obtained that there exists a point such that no orbit pass through it. Based on the correspondence between the two cases before and after the transformation (Lemma 3.2), it is achieved that the original system admits no Lagrangian torus with the rotation vector $\omega$.

The paper is outlined as follows. In Section 2, we prove Theorem 1.1 for a special case with the rotation vector having a small first coordinate and a suitable large second coordinate. In Section 3, the transformation of the coordinates is given and based on that, we complete the proof of Theorem 1.1 . 


\section{Destruction of Lagrangian torus with a special rotation vector}

After a suitable transformation of coordinates, the problem with a given rotation vector $\omega$ can be reduced to the case with the rotation vector having a small first coordinate and a suitable large second coordinate. In this section, we will prove Theorem 1.1 for that case.

The Hamiltonian function we consider here is nearly integrable

$$
H_{n}(q, p)=H_{0}(p)-P_{n}(q),
$$

where $(q, p) \in \mathbb{T}^{d} \times \mathbb{R}^{d}$. For the sake of simplicity, we assume

$$
H_{0}(p)=\frac{1}{2}|p|^{2} .
$$

Since $H_{n}$ is strictly convex with respect to $p$, by the Legendre transformation, the Lagrangian function corresponding to $H_{n}$ is

$$
L_{n}(q, \dot{q})=\frac{1}{2}|\dot{q}|^{2}+P_{n}(q),
$$

where $\dot{q}=\frac{\partial H_{0}}{\partial p}$ and $q=\left(q_{1}, q_{2}, \ldots, q_{d}\right)$.

Let

$$
P_{n}(q)=\frac{1}{n^{a}}\left(1-\cos q_{1}\right)+v_{n}\left(q_{1}, q_{2}\right),
$$

where $a$ is a positive constant independent of $n$. For the rotation vector $\omega=$ $\left(\omega_{1}, \ldots, \omega_{d}\right), v_{n}\left(q_{1}, q_{2}\right)$ is constructed as follow

$$
\left\{\begin{array}{l}
v_{n} \text { is } 2 \pi \text {-periodic, } \\
\operatorname{supp} v_{n} \cap\{[0,2 \pi] \times[-\pi, \pi]\}=B_{R_{n}}\left(q^{*}\right), \\
\max _{\left(q_{1}, q_{2}\right) \in[0,2 \pi] \times[-\pi, \pi]} v_{n}=v_{n}\left(q^{*}\right)=\left|\omega_{1}\right|^{s}, \\
\left\|v_{n}\right\|_{C^{r}} \sim\left|\omega_{1}\right|^{s^{\prime}},
\end{array}\right.
$$

where $R_{n}=\frac{\left|\omega_{1}\right|}{n^{2}}, q^{*}=(\pi, 0)$ and we require $s^{\prime}>4$, it can be satisfied for $s$ suitable large. $f \sim g$ means that $\frac{1}{C} g<f<C g$ holds for some constant $C>1$,

For (2.1), we have the following lemma.

Lemma 2.1 For n large enough, the Hamiltonian flow generated by $H_{n}(q, p)$ does not admit any Lagrangian torus with rotation vector $\omega=\left(\omega_{1}, \ldots, \omega_{d}\right)$ satisfying

$$
\left|\omega_{1}\right|<n^{-\frac{a}{2}-\epsilon} \quad \text { and } \quad\left|\omega_{2}\right| \sim n,
$$

where $\epsilon>0$ is independent of $n$.

Lemma 2.1 will be proved with variational method in Subsection 2.3. First of all, we put it into the Lagrangian formalism. Let $\sigma_{n}=n^{-a}$. The Lagrangian function corresponding to $(2.2)$ is

$$
L_{n}\left(q_{1}, Q, \dot{q}_{1}, \dot{Q}\right)=\frac{1}{2}|\dot{Q}|^{2}+\frac{1}{2}\left|\dot{q}_{1}\right|^{2}+\sigma_{n}\left(1-\cos \left(q_{1}\right)\right)+v_{n}\left(q_{1}, q_{2}\right),
$$

where $Q=\left(q_{2}, \ldots, q_{d}\right) . \quad L_{n}\left(q_{1}, Q, \dot{q}_{1}, \dot{Q}\right)$ consists of one pendulum, a rotator with $d-1$ degrees of freedom and a perturbation coupling of them. The pendulum has the following Lagrangian function

$$
A_{n}\left(q_{1}, \dot{q}_{1}\right)=\frac{1}{2}\left|\dot{q}_{1}\right|^{2}+\sigma_{n}\left(1-\cos \left(q_{1}\right)\right),
$$


which corresponds to the Hamiltonian via Legendre transformation

$$
h_{n}\left(q_{1}, p_{1}\right)=\frac{1}{2}\left|p_{1}\right|^{2}-\sigma_{n}\left(1-\cos q_{1}\right) .
$$

\subsection{The action of the simple pendulum}

Each solution of the Lagrangian equation determined by $A_{n}$, denoted by $q_{1}(t)$, determines an orbit $\left(q_{1}(t), p_{1}(t)\right)$ of the Hamiltonian flow generated by $h_{n}$. Each orbit stays in certain energy level set $\left(q_{1}, p_{1}\right) \in h_{n}^{-1}(e)$. Under the boundary condition that $q_{1}\left(t_{0}\right)=0, q_{1}\left(t_{1}\right)=\pi$ ( or $q_{1}\left(t_{1}\right)=\pi, q_{1}\left(t_{2}\right)=2 \pi$ ), there is a unique correspondence between $t_{1}-t_{0}$ and the energy, denoted by $e\left(t_{1}-t_{0}\right)$, such that the determined orbit stays in the energy level set $h_{n}^{-1}\left(e\left(t_{1}-t_{0}\right)\right)$. More precisely, we have the following lemma.

Lemma 2.2 Let $\bar{q}_{1}$ be the solution of $A_{n}$ on $\left(t_{0}, \bar{t}_{1}\right)$ satisfying the boundary conditions

$$
\left\{\begin{array}{l}
\bar{q}_{1}\left(t_{0}\right)=0, \\
\bar{q}_{1}\left(\bar{t}_{1}\right)=\pi,
\end{array}\right.
$$

$e\left(\bar{t}_{1}-t_{0}\right)$ be the energy of $\bar{q}_{1}$, i.e. $\left(\bar{q}_{1}, \bar{p}_{1}\right) \in h_{n}^{-1}\left(e\left(\bar{t}_{1}-t_{0}\right)\right)$ and $\omega_{1}$ be the average speed of $\bar{q}_{1}$ on $\left(t_{0}, \bar{t}_{1}\right)$. If $\left|\omega_{1}\right|<n^{-\frac{a}{2}-\epsilon}$, then

$$
e\left(\bar{t}_{1}-t_{0}\right) \sim \sigma_{n} \exp \left(-\frac{C \sqrt{\sigma_{n}}}{\left|\omega_{1}\right|}\right)
$$

where $\sigma_{n}=n^{-a}$.

Proof By the definition, we have

$$
\frac{1}{2}\left|\dot{\bar{q}}_{1}\right|^{2}-\sigma_{n}\left(1-\cos \left(\bar{q}_{1}\right)\right)=e\left(\bar{t}_{1}-t_{0}\right)
$$

hence

$$
\left|\dot{\bar{q}}_{1}\right|=\sqrt{2\left(e\left(\bar{t}_{1}-t_{0}\right)+\sigma_{n}\left(1-\cos \left(\bar{q}_{1}\right)\right)\right)} .
$$

Since $\bar{q}_{1}\left(t_{0}\right)=0$, then

$$
e\left(\bar{t}_{1}-t_{0}\right)=\frac{1}{2}\left|\dot{\bar{q}}_{1}\left(t_{0}\right)\right|^{2} \leq \frac{1}{2}\left|\omega_{1}\right|^{2} \sim n^{-a-2 \epsilon},
$$

which together with $\sigma_{n}=n^{-a}$ implies that $\sigma_{n} / e\left(\bar{t}_{1}-t_{0}\right) \gg 1$. Since the average speed of $\bar{q}_{1}$ is $\omega_{1}$, by a direct calculation, we have

$$
\frac{\pi}{\left|\omega_{1}\right|}=\int_{t_{0}}^{\bar{t}_{1}} d t=\int_{0}^{\pi} \frac{d \bar{q}_{1}}{\sqrt{2\left(e\left(\bar{t}_{1}-t_{0}\right)+\sigma_{n}\left(1-\cos \left(\bar{q}_{1}\right)\right)\right)}} \sim \frac{1}{\sqrt{\sigma_{n}}} \ln \left(\frac{\sigma_{n}}{e\left(\bar{t}_{1}-t_{0}\right)}\right),
$$

moreover,

$$
e\left(\bar{t}_{1}-t_{0}\right) \sim \sigma_{n} \exp \left(-\frac{C \sqrt{\sigma_{n}}}{\left|\omega_{1}\right|}\right),
$$

which completes the proof of Lemma 2.2 . 
Remark 2.3 It is easy to see that Lemma 2.2 also holds for

$$
\left\{\begin{array}{l}
\bar{q}_{1}\left(\bar{t}_{1}\right)=\pi \\
\bar{q}_{1}\left(t_{2}\right)=2 \pi
\end{array}\right.
$$

Moreover, we denote the difference of time by $\Delta t$, then $e(\Delta t)$ is an decreasing function with respect to $\Delta t \geq 0$ for any $\omega_{1} \in \mathbb{R}$.

The following lemma implies that the actions along orbits in the neighborhood of the separatrix of the pendulum does not change too much with respect to a small change in speed (time).

Lemma 2.4 Let $\bar{t}_{1}, \tilde{t}_{1} \in\left[t_{0}, t_{2}\right]$. Let $\bar{q}_{1}(t)$ be a solution of $A_{n}$ on $\left(t_{0}, \bar{t}_{1}\right)$ and $\left(\bar{t}_{1}, t_{2}\right)$ with boundary conditions respectively

$$
\left\{\begin{array} { l } 
{ \overline { q } _ { 1 } ( t _ { 0 } ) = 0 , } \\
{ \overline { q } _ { 1 } ( \overline { t } _ { 1 } ) = \pi , }
\end{array} \quad \left\{\begin{array}{l}
\bar{q}_{1}\left(\bar{t}_{1}\right)=\pi \\
\bar{q}_{1}\left(t_{2}\right)=2 \pi
\end{array}\right.\right.
$$

and let $\tilde{q}_{1}(t)$ be a solution of $A_{n}$ on $\left(t_{0}, \tilde{t}_{1}\right)$ and $\left(\tilde{t}_{1}, t_{2}\right)$ with boundary conditions respectively

$$
\left\{\begin{array} { l } 
{ \tilde { q } _ { 1 } ( t _ { 0 } ) = 0 , } \\
{ \tilde { q } _ { 1 } ( \tilde { t } _ { 1 } ) = \pi , }
\end{array} \quad \left\{\begin{array}{l}
\tilde{q}_{1}\left(\tilde{t}_{1}\right)=\pi \\
\tilde{q}_{1}\left(t_{2}\right)=2 \pi
\end{array}\right.\right.
$$

Let $\bar{\omega}_{1}^{\prime}$ and $\bar{\omega}_{1}^{\prime \prime}$ be the average speed of $\bar{q}_{1}$ on $\left(t_{0}, \bar{t}_{1}\right)$ and $\left(\bar{t}_{1}, t_{2}\right)$ respectively. Let $\tilde{\omega}_{1}^{\prime}$ and $\tilde{\omega}_{1}^{\prime \prime}$ be the average speed of $\tilde{q}_{1}$ on $\left(t_{0}, \tilde{t}_{1}\right)$ and $\left(\tilde{t}_{1}, t_{2}\right)$ respectively. We set

$$
\left|\omega_{1}\right|=\max \left\{\left|\bar{\omega}_{1}^{\prime}\right|,\left|\bar{\omega}_{1}^{\prime \prime}\right|,\left|\tilde{\omega}_{1}^{\prime}\right|,\left|\tilde{\omega}_{1}^{\prime \prime}\right|\right\} .
$$

If $\left|\omega_{1}\right|<n^{-\frac{a}{2}-\epsilon}$, then

$$
\left|\int_{t_{0}}^{t_{2}} A_{n}\left(\bar{q}_{1}, \dot{\bar{q}}_{1}\right) d t-\int_{t_{0}}^{t_{2}} A_{n}\left(\tilde{q}_{1}, \dot{\tilde{q}}_{1}\right) d t\right| \leq C_{1}\left|\bar{t}_{1}-\tilde{t}_{1}\right| \sigma_{n} \exp \left(-\frac{C_{2} \sqrt{\sigma_{n}}}{\left|\omega_{1}\right|}\right) .
$$

Proof The proof follows the similar idea of Lemma 4 in $\left[\mathrm{Be}\right.$. Let $q_{1}(t)$ be a solution of $A_{n}$ on $\left(t_{0}, t_{1}\right)$ and $\left(t_{1}, t_{2}\right)$ with boundary conditions respectively

$$
\left\{\begin{array} { l } 
{ q _ { 1 } ( t _ { 0 } ) = 0 , } \\
{ q _ { 1 } ( t _ { 1 } ) = \pi , }
\end{array} \quad \left\{\begin{array}{l}
q_{1}\left(t_{1}\right)=\pi \\
q_{1}\left(t_{2}\right)=2 \pi
\end{array}\right.\right.
$$

We consider the function

$$
\begin{aligned}
L\left(t_{1}\right)= & \int_{t_{0}}^{t_{1}} A_{n}\left(q_{1}, \dot{q}_{1}\right) d t+\int_{t_{1}}^{t_{2}} A_{n}\left(q_{1}, \dot{q}_{1}\right) d t \\
= & \int_{0}^{\pi} \sqrt{2\left(e\left(t_{1}-t_{0}\right)+V\left(q_{1}\right)\right)} d q_{1}-e\left(t_{1}-t_{0}\right)\left(t_{1}-t_{0}\right) \\
& +\int_{\pi}^{2 \pi} \sqrt{2\left(e\left(t_{2}-t_{1}\right)+V\left(q_{1}\right)\right)} d q_{1}-e\left(t_{2}-t_{1}\right)\left(t_{2}-t_{1}\right),
\end{aligned}
$$

where

$$
V\left(q_{1}\right)=\sigma_{n}\left(1-\cos \left(q_{1}\right)\right)
$$


and $e(\Delta t)$ denotes the energy of the orbit of the pendulum moving half a turn in time $\Delta t$. The quantity $e(\Delta t)$ is differentiable with respect to $\Delta t$, then

$$
\begin{aligned}
\frac{d L\left(t_{1}\right)}{d t_{1}}= & \int_{0}^{\pi} \frac{\dot{e}\left(t_{1}-t_{0}\right)}{\sqrt{2\left(e\left(t_{1}-t_{0}\right)+V\left(q_{1}\right)\right)}} d q_{1}-\dot{e}\left(t_{1}-t_{0}\right)\left(t_{1}-t_{0}\right) \\
& -e\left(t_{1}-t_{0}\right)-\int_{\pi}^{2 \pi} \frac{\dot{e}\left(t_{2}-t_{1}\right)}{\sqrt{2\left(e\left(t_{2}-t_{1}\right)+V\left(q_{1}\right)\right)}} d q_{1} \\
& +\dot{e}\left(t_{2}-t_{1}\right)\left(t_{2}-t_{1}\right)+e\left(t_{2}-t_{1}\right), \\
= & \int_{t_{0}}^{t_{1}} \dot{e}\left(t_{1}-t_{0}\right) d t-\dot{e}\left(t_{1}-t_{0}\right)\left(t_{1}-t_{0}\right)-e\left(t_{1}-t_{0}\right) \\
& -\int_{t_{1}}^{t_{2}} \dot{e}\left(t_{2}-t_{1}\right) d t+\dot{e}\left(t_{2}-t_{1}\right)\left(t_{2}-t_{1}\right)+e\left(t_{2}-t_{1}\right), \\
= & e\left(t_{2}-t_{1}\right)-e\left(t_{1}-t_{0}\right) .
\end{aligned}
$$

Thus, we have

$$
\left|\frac{d L\left(t_{1}\right)}{d t_{1}}\right| \leq\left|e\left(t_{2}-t_{1}\right)\right|+\left|e\left(t_{1}-t_{0}\right)\right| .
$$

Integrate from $\bar{t}_{1}$ to $\tilde{t}_{1}$ and from (2.7), it follows that

$$
\left|\int_{t_{0}}^{t_{2}} A_{n}\left(\bar{q}_{1}, \dot{\bar{q}}_{1}\right) d t-\int_{t_{0}}^{t_{2}} A_{n}\left(\tilde{q}_{1}, \dot{\tilde{q}}_{1}\right) d t\right| \leq C_{1}\left|\bar{t}_{1}-\tilde{t}_{1}\right| \sigma_{n} \exp \left(-\frac{C_{2} \sqrt{\sigma_{n}}}{\left|\omega_{1}\right|}\right),
$$

which completes the proof of Lemma 2.4.

Remark 2.5 Since (2.9) holds for any $\omega_{1} \in \mathbb{R}$, combining with the monotonicity of $e(\Delta t)$, it is easy to draw the following figure for $L(t) . L(t)$ is decreasing for $t \in\left(t_{0}, \frac{t_{0}+t_{2}}{2}\right]$ and increasing for $t \in\left[\frac{t_{0}+t_{2}}{2}, t_{2}\right)$. See Fig. 1

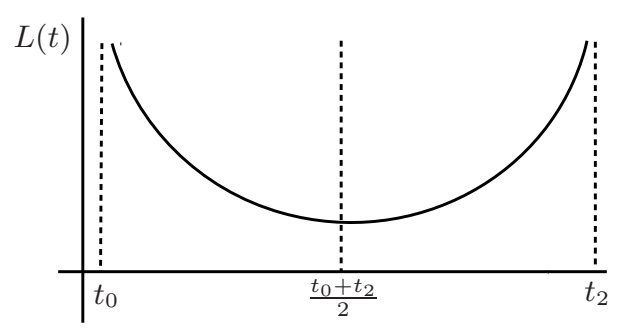

Figure 1: $L(t)$

\subsection{The velocity of the action minimizing orbit}

Once the function $q_{1}(t)$ is fixed, the function $Q(t)$ is the solution of the EulerLagrange equation with the non autonomous Lagrangian

$$
\hat{L}_{n}(Q(t), \dot{Q}(t), t)=\frac{1}{2}|\dot{Q}(t)|^{2}+v_{n}\left(q_{1}(t), q_{2}(t)\right),
$$

where $Q(t)=\left(q_{2}(t), \ldots, q_{d}(t)\right)$. The first derivative of $\hat{L}_{n}(Q(t), \dot{Q}(t), t)$ with respect to $Q$ is easy to obtained as follows:

$$
\frac{\partial \hat{L}_{n}}{\partial q_{2}}=\frac{\partial v_{n}}{\partial q_{2}}\left(q_{1}(t), q_{2}(t)\right) \quad \text { and } \quad \frac{\partial \hat{L}_{n}}{\partial q_{i}}=0 \quad \text { for } \quad i=3, \ldots, d .
$$


From the construction of $v_{n}$ (see (2.3) $)$, we have $\left\|v_{n}\right\|_{C^{r}} \sim\left|\omega_{1}\right|^{s^{\prime}}$. It follows from $s^{\prime}>4$ that

$$
\left|\frac{\partial \hat{L}_{n}}{\partial q_{i}}\right| \leq C\left|\omega_{1}\right|^{4} \quad \text { for } \quad i=2, \ldots, d . .
$$

Based on the periodicity of $\hat{L}_{n}(Q(t), \dot{Q}(t), t)$, by Lemma 2 of [BK], we have the following estimate.

Lemma 2.6 Let $\left(q_{1}(t), Q(t)\right)$ be the action minimizing orbit of $L_{n}$ with rotation vector $\omega$, then for any $t^{\prime}, t^{\prime \prime} \in \mathbb{R}$ and $t \in\left[t^{\prime}, t^{\prime \prime}\right]$ we have

$$
\left|\dot{Q}(t)-\frac{Q\left(t^{\prime \prime}\right)-Q\left(t^{\prime}\right)}{t^{\prime \prime}-t^{\prime}}\right| \leq C\left|\omega_{1}\right|^{2}
$$

\subsection{Proof of Lemma 2.1}

If the Hamiltonian flow generated by $H_{n}$ admits a Lagrangian torus with nonresonant rotation vector $\omega$, then there is a unique minimal curve $q(t)$ with rotation vector $\omega$ passing through each $x \in \mathbb{T}^{d}$ since the Lagrangian torus is a graph. By [H4], each orbit on the Lagrangian torus is an action minimizing curve. Hence, it is sufficient to prove the existence of some point in $\mathbb{T}^{d}$ where no minimal curve passes through.

Indeed, any minimal curve does not pass through the subspace $(\pi, 0) \times \mathbb{T}^{d-2}$, which implies Lemma 2.1. Let us assume the contrary, namely, there exists $\bar{t}_{1}$ such that

$$
q_{1}\left(\bar{t}_{1}\right)=\pi, \quad q_{2}\left(\bar{t}_{1}\right)=0,
$$

where $q(t)=\left(q_{1}, q_{2}, \ldots, q_{d}\right)(t)$ is a minimal curve in the universal covering space $\mathbb{R}^{d}$. Because of $\omega_{1} \neq 0$, there exist $t_{0}$ and $t_{2}$ such that

$$
q_{1}\left(t_{0}\right)=0, \quad q_{1}\left(t_{2}\right)=2 \pi .
$$

Obviously, $t_{0}<\bar{t}_{1}<t_{2}$.

Claim $t_{2}-t_{0} \sim\left|\omega_{1}\right|^{-1}$.

Proof We assume by contradiction that $t_{2}-t_{0} \sim\left|\bar{\omega}_{1}\right|^{-1}$ and without loss of generality, $\bar{\omega}_{1}=o\left(\left|\omega_{1}\right|\right)$ as $n \rightarrow \infty$. Moreover, for $\left[t_{0}, \bar{t}_{1}\right]$ and $\left[\bar{t}_{1}, t_{2}\right]$, there exists at least one interval with the length not less than $\left|\bar{\omega}_{1}\right|^{-1}$, otherwise it is contradicted by $t_{2}-t_{0} \sim\left|\bar{\omega}_{1}\right|^{-1}$. Without loss of generality, one can assume that $t_{2}-\bar{t}_{1}>\left|\bar{\omega}_{1}\right|^{-1}$.

From the definition of rotation vector, $\lim _{t \rightarrow \infty}\left(q_{1}(t)-q_{1}\left(\bar{t}_{1}\right)\right) /\left(t-\bar{t}_{1}\right)=\omega_{1}$, we have that for any $\epsilon>0$, we can find $t_{N}>t_{2}$ such that

$$
\left|\frac{q_{1}\left(t_{N}\right)-q_{1}\left(\bar{t}_{1}\right)}{t_{N}-\bar{t}_{1}}-\omega_{1}\right| \leq \epsilon \text { and } q_{1}\left(t_{N}\right)=N \pi
$$

where $N$ depends on $n$ and $N \gg 2$. Then we have

$$
t_{N}-\bar{t}_{1} \sim \frac{N \pi}{\left|\omega_{1}\right|}
$$

We choose a sequence of times $t_{2}<\ldots<t_{N}$ satisfying $q_{1}\left(t_{i}\right)=i \pi$ for $i \in\{2, \ldots, N\}$. Moreover, it follows from Pigeon hole principle that there exists $j \in\{2, \ldots, N-1\}$ such that

$$
t_{j+1}-t_{j}<\left|\bar{\omega}_{1}\right|^{-1}
$$


where we consider the case $q_{1}\left(t_{j}\right) \bmod 2 \pi=0$ and $q_{1}\left(t_{j+1}\right) \bmod 2 \pi=\pi$, the other case is similar. Let $q_{1}\left(\bar{t}_{1}^{\prime}\right)=\pi+R_{n}$ and $q_{1}\left(t_{j+1}^{\prime}\right)=(j+1) \pi-R_{n}$, where $R_{n}$ is the radius of the support of $v_{n}$. By the minimality of $q(t)$, there exist positive constants $C^{\prime}$ and $C^{\prime \prime}$ such that $t_{2}-\bar{t}_{1}^{\prime}>C^{\prime}\left|\bar{\omega}_{1}\right|^{-1}$ and $t_{j+1}^{\prime}-t_{j}<C^{\prime \prime}\left|\bar{\omega}_{1}\right|^{-1}$. Hence, we can substitute $\left.q_{1}(t)\right|_{\left[\bar{t}_{1}^{\prime}, t_{2}\right]}$ and $\left.q_{1}(t)\right|_{\left[t_{j}, t_{j+1}^{\prime}\right]}$ by the orbits $\hat{q}_{1^{\prime}}(t), \hat{q}_{1^{\prime \prime}}(t)$ of the pendulum $A_{n}$ (see (2.5) ) with more uniform motion. Correspondingly, we substitute $\left.q_{2}(t)\right|_{\left[\bar{t}_{1}^{\prime}, t_{2}\right]}$ and $\left.q_{2}(t)\right|_{\left[t_{j}, t_{j+1}^{\prime}\right]}$ by the orbits $\hat{q}_{2^{\prime}}(t), \hat{q}_{2^{\prime \prime}}(t)$ of uniform linear motion. From Remark 2.5, the action of $A_{n}$ will decrease based on the more uniform motion of the pendulum. Since the motion of $\hat{q}_{2^{\prime}}(t), \hat{q}_{2^{\prime \prime}}(t)$ are uniform linear, then the actions of $\frac{1}{2}\left|\dot{\hat{q}}_{2^{\prime}}(t)\right|^{2}$ and $\frac{1}{2}\left|\dot{\hat{q}}_{2^{\prime \prime}}(t)\right|^{2}$ are not greater than the action of $\frac{1}{2}\left|\dot{q}_{2}(t)\right|^{2}$. More precisely, similar to Be], we denote

$$
T=\frac{1}{2}\left(t_{2}-\bar{t}_{1}^{\prime}+t_{j+1}^{\prime}-t_{j}\right) .
$$

Let $s_{j}$ be the closest time to $t_{j+1}^{\prime}-T$ such that $q_{2}\left(s_{j}\right)-q_{2}\left(t_{j}\right)=l$ where $l \in 2 \pi \mathbb{Z}$. Since $\left|\omega_{2}\right| \sim n$, it follows from Lemma 2.6 that $\left|t_{j+1}^{\prime}-T-s_{j}\right| \leq C / n$. Let $s_{2}=s_{j}-\left(t_{j}-t_{2}\right)$. Then $\hat{q}_{1^{\prime}}(t)$ and $\hat{q}_{1^{\prime \prime}}(t)$ are the solutions of $A_{n}$ on $\left(\bar{t}_{1}^{\prime}, s_{2}\right)$ and on $\left(s_{j}, t_{j+1}^{\prime}\right)$ with boundary conditions respectively

$$
\left\{\begin{array} { l } 
{ \hat { q } _ { 1 ^ { \prime } } ( \overline { t } _ { 1 } ^ { \prime } ) = q _ { 1 } ( \overline { t } _ { 1 } ^ { \prime } ) , } \\
{ \hat { q } _ { 1 ^ { \prime } } ( s _ { 2 } ) = q _ { 1 } ( t _ { 2 } ) = \pi , }
\end{array} \quad \left\{\begin{array}{l}
\hat{q}_{1^{\prime \prime}}\left(s_{j}\right)=q_{1}\left(t_{j}\right)=j \pi, \\
\hat{q}_{1^{\prime \prime}}\left(t_{j+1}^{\prime}\right)=q_{1}\left(t_{j+1}^{\prime}\right) .
\end{array}\right.\right.
$$

Correspondingly, we construct $\hat{q}_{2^{\prime}}(t)$ and $\hat{q}_{2^{\prime \prime}}(t)$ respectively

$$
\begin{cases}\hat{q}_{2^{\prime}}(t)=q_{2}\left(\bar{t}_{1}^{\prime}\right)+\frac{q_{2}\left(t_{2}\right)-q_{2}\left(\bar{t}_{1}^{\prime}\right)+l}{s_{2}-\bar{t}_{1}^{\prime}}\left(t-\bar{t}_{1}^{\prime}\right), & t \in\left[\bar{t}_{1}^{\prime}, s_{2}\right], \\ \hat{q}_{2^{\prime \prime}}(t)=q_{2}\left(t_{j}\right)+l+\frac{q_{2}\left(t_{j+1}^{\prime}\right)-q_{2}\left(t_{j}\right)-l}{t_{j+1}^{\prime}-s_{j}}\left(t-s_{j}\right), & t \in\left[s_{j}, t_{j+1}^{\prime}\right] .\end{cases}
$$

Moreover, we set

$$
\begin{gathered}
\hat{q}_{1}:=\left.\left.q_{1}(t)\right|_{\left[\bar{t}_{1}, \bar{t}_{1}^{\prime}\right]} * \hat{q}_{1^{\prime}}(t)\right|_{\left.\left.\left.{\left[t_{1}^{\prime}, s_{2}\right]}_{1} * q_{1}\left(t-s_{2}+t_{2}\right)\right|_{\left[s_{2}, s_{j}\right]} * \hat{q}_{1^{\prime \prime}}(t)\right|_{\left[s_{j}, t_{j+1}^{\prime}\right]} * q_{1}(t)\right|_{\left[t_{j+1}^{\prime}, t_{j+1}\right]},} \hat{q}_{2}:=\left.\left.\left.\left.q_{2}(t)\right|_{\left[\bar{t}_{1}, \bar{t}_{1}^{\prime}\right]} * \hat{q}_{2^{\prime}}(t)\right|_{\left[\bar{t}_{1}^{\prime}, s_{2}\right]} *\left(\left.q_{2}\left(t-s_{2}+t_{2}\right)\right|_{\left[s_{2}, s_{j}\right]}+l\right) * \hat{q}_{2^{\prime \prime}}(t)\right|_{\left[s_{j}, t_{j+1}^{\prime}\right]} * q_{2}(t)\right|_{\left[t_{j+1}^{\prime}, t_{j+1}\right]},
\end{gathered}
$$

where $*$ denotes the juxtaposition of curves. Let $\hat{Q}=\left(\hat{q}_{2}, q_{3}, \ldots, q_{d}\right)$ and $Q=$ $\left(q_{2}, q_{3}, \ldots, q_{d}\right)$. Then we have

$$
\int_{\bar{t}_{1}}^{t_{N}} L_{n}\left(\hat{q}_{1}, \hat{Q}, \dot{\hat{q}}_{1}, \dot{\hat{Q}}\right) d t<\int_{\bar{t}_{1}}^{t_{N}} L_{n}\left(q_{1}, Q, \dot{q}_{1}, \dot{Q}\right) d t
$$

which is contradicted by the minimality of $q(t)$. Hence, $t_{2}-t_{0} \sim\left|\omega_{1}\right|^{-1}$.

Let $\tilde{t}_{1}$ be the last time before $\bar{t}_{1}$ or the first time after $\bar{t}_{1}$ such that

$$
\left|q_{2}\left(\tilde{t}_{1}\right)-q_{2}\left(\bar{t}_{1}\right)\right|=\pi .
$$

Since $\left|\omega_{1}\right|$ is small enough for $n$ large enough and $\left|\omega_{2}\right| \sim n$, by Lemma 2.6, we have that for $t \in\left[t_{0}, t_{2}\right],\left|\dot{q}_{2}(t)\right| \sim n$,

$$
\left|\tilde{t}_{1}-\bar{t}_{1}\right| \leq \frac{C_{0}}{n} .
$$


Without loss of generality, one can assume $\omega_{1}>0$ and $\omega_{2}>0$. Consider a solution $\tilde{q}_{1}$ of $A_{n}$ on $\left(t_{0}, \tilde{t}_{1}\right)$ and on $\left(\tilde{t}_{1}, t_{2}\right)$ with boundary conditions respectively

$$
\left\{\begin{array} { l } 
{ \tilde { q } _ { 1 } ( t _ { 0 } ) = q _ { 1 } ( t _ { 0 } ) = 0 , } \\
{ \tilde { q } _ { 1 } ( \tilde { t } _ { 1 } ) = q _ { 1 } ( \overline { t } _ { 1 } ) = \pi , }
\end{array} \quad \left\{\begin{array}{l}
\tilde{q}_{1}\left(\tilde{t}_{1}\right)=q_{1}\left(\bar{t}_{1}\right)=\pi, \\
\tilde{q}_{1}\left(t_{2}\right)=q_{1}\left(t_{2}\right)=2 \pi .
\end{array}\right.\right.
$$

Since $q$ is assumed to be a minimal curve, we have

$$
\int_{t_{0}}^{t_{2}} L_{n}\left(\tilde{q}_{1}, Q, \dot{\tilde{q}}_{1}, \dot{Q}\right) d t-\int_{t_{0}}^{t_{2}} L_{n}\left(q_{1}, Q, \dot{q}_{1}, \dot{Q}\right) d t \geq 0
$$

See Fig. 2 , where $x_{1}=\left(q_{1}\left(\bar{t}_{1}\right), q_{2}\left(\bar{t}_{1}\right)\right)=(\pi, 0), x_{0}=\left(q_{1}\left(t_{0}\right), q_{2}\left(t_{0}\right)\right)=\left(0, q_{2}\left(t_{0}\right)\right)$, $x_{2}=\left(q_{1}\left(t_{2}\right), q_{2}\left(t_{2}\right)\right)=\left(2 \pi, q_{2}\left(t_{2}\right)\right), \tilde{x}_{1}^{\prime}=(\pi,-\pi)$ and $\tilde{x}_{1}^{\prime \prime}=(\pi, \pi)$.

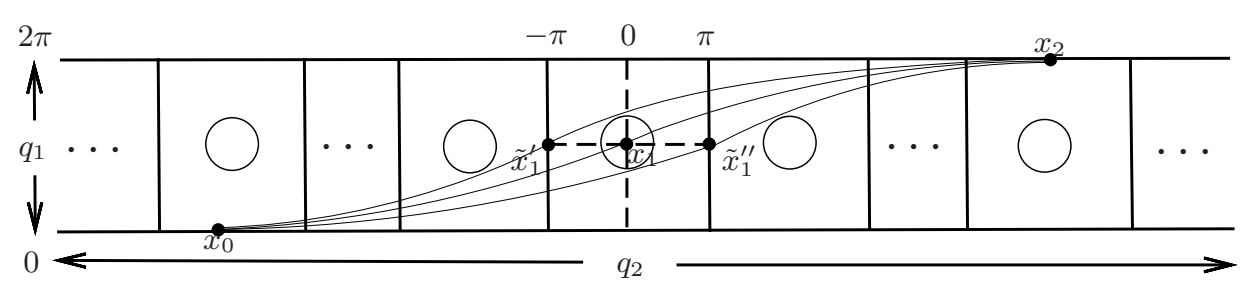

Figure 2: The projections of the curves $\left(q_{1}(t), Q(t)\right)$ and $\left(\tilde{q}_{1}(t), Q(t)\right)$ on $[0,2 \pi] \times \mathbb{R}$

$\left(\tilde{q}_{1}(t), q_{2}(t)\right)$ passes through the point $\tilde{x}_{1}^{\prime}$ or $\tilde{x}_{1}^{\prime \prime}$. Thus, by the construction of $L_{n}$, we obtain from (2.13) that

$$
\int_{t_{0}}^{t_{2}} A_{n}\left(\tilde{q}_{1}, \dot{\tilde{q}}_{1}\right) d t-\int_{t_{0}}^{t_{2}} A_{n}\left(q_{1}, \dot{q}_{1}\right) d t \geq \int_{t_{0}}^{t_{2}} v_{n}\left(q_{1}, q_{2}\right) d t-\int_{t_{0}}^{t_{2}} v_{n}\left(\tilde{q}_{1}, q_{2}\right) d t .
$$

By the definition of $v_{n}$ as (2.3), we have the following claim:

Claim $\left(\tilde{q}_{1}(t), q_{2}(t)\right) \notin \operatorname{supp} v_{n}$ for $t \in\left(t_{0}, t_{2}\right)$.

Proof We assume by contradiction that there would exist $\hat{t}$ such that $\left(\tilde{q}_{1}(\hat{t}), q_{2}(\hat{t})\right) \in$ $\operatorname{supp} v_{n}$, without loss of generality, one can assume $\hat{t}>\tilde{t}_{1}$. By Lemma 2.6. for any $t \in\left[\tilde{t}_{1}, \hat{t}\right]$,

$$
\dot{q}_{2}(t) \leq C_{1} n
$$

hence,

$$
\hat{t}-\tilde{t}_{1} \geq \frac{C_{2}}{n},
$$

where $C_{1}, C_{2}$ are constants independent of $n$.

Let $\tilde{\omega}_{1}^{\prime}$ and $\tilde{\omega}_{1}^{\prime \prime}$ be the average speed of $\tilde{q}_{1}$ on $\left(t_{0}, \tilde{t}_{1}\right)$ and $\left(\tilde{t}_{1}, t_{2}\right)$ respectively, then

$$
\frac{2 \pi}{\left|\omega_{1}\right|}=\frac{\pi}{\left|\tilde{\omega}_{1}^{\prime}\right|}+\frac{\pi}{\left|\tilde{\omega}_{1}^{\prime \prime}\right|}
$$

hence,

$$
\left|\tilde{\omega}_{1}^{\prime}\right| \geq \frac{1}{2}\left|\omega_{1}\right| \quad \text { and } \quad\left|\tilde{\omega}_{1}^{\prime \prime}\right| \geq \frac{1}{2}\left|\omega_{1}\right|,
$$

which together with the Euler-Lagrange equation of $\tilde{q}_{1}(t)$ implies that for any $t \in$ $\left[\tilde{t}_{1}, \hat{t}\right]$,

$$
\dot{\tilde{q}}_{1}(t) \geq C_{3}\left|\omega_{1}\right|
$$


consequently

$$
\left|\tilde{q}_{1}(\hat{t})-\tilde{q}_{1}\left(\tilde{t}_{1}\right)\right| \geq C_{4} \frac{\left|\omega_{1}\right|}{n},
$$

where $R_{n}$ is the radius of the support of $v_{n}$. Since $R_{n}=\frac{\left|\omega_{1}\right|}{n^{2}}$, then we have

$$
\left|\tilde{q}_{1}(\hat{t})-\tilde{q}_{1}\left(\tilde{t}_{1}\right)\right|>R_{n}
$$

which is contradicted by the assumption $\left(\tilde{q}_{1}(\hat{t}), q_{2}(\hat{t})\right) \in \operatorname{supp} v_{n}$.

Hence, we have

$$
\int_{t_{0}}^{t_{2}} v_{n}\left(q_{1}, q_{2}\right) d t-\int_{t_{0}}^{t_{2}} v_{n}\left(\tilde{q}_{1}, q_{2}\right) d t=\int_{t_{0}}^{t_{2}} v_{n}\left(q_{1}, q_{2}\right) d t
$$

By the construction of $v_{n}$ and the minimality of $\left(q_{1}, Q\right)$, a simple calculation shows

$$
\int_{t_{0}}^{t_{2}} v_{n}\left(q_{1}, q_{2}\right) d t \geq\left|\omega_{1}\right|^{\lambda}
$$

where $\lambda$ is a positive constant. Consequently, if follows from (2.14) that

$$
\int_{t_{0}}^{t_{2}} A_{n}\left(\tilde{q}_{1}, \dot{\tilde{q}}_{1}\right) d t-\int_{t_{0}}^{t_{2}} A_{n}\left(q_{1}, \dot{q}_{1}\right) d t \geq\left|\omega_{1}\right|^{\lambda}
$$

On the other hand, consider a solution $\bar{q}_{1}$ of $A_{n}$ on $\left(t_{0}, \bar{t}_{1}\right)$ and on $\left(\bar{t}_{1}, t_{2}\right)$ with boundary conditions respectively

$$
\left\{\begin{array} { l } 
{ \overline { q } _ { 1 } ( t _ { 0 } ) = q _ { 1 } ( t _ { 0 } ) = 0 , } \\
{ \overline { q } _ { 1 } ( \overline { t } _ { 1 } ) = q _ { 1 } ( \overline { t } _ { 1 } ) = \pi , }
\end{array} \quad \left\{\begin{array}{l}
\bar{q}_{1}\left(\bar{t}_{1}\right)=q_{1}\left(\bar{t}_{1}\right)=\pi, \\
\bar{q}_{1}\left(t_{2}\right)=q_{1}\left(t_{2}\right)=2 \pi .
\end{array}\right.\right.
$$

For $t \in\left(t_{0}, \bar{t}_{1}\right)$ and $\left(\bar{t}_{1}, t_{2}\right)$ respectively, the action of $A_{n}$ achieves the minima along $\bar{q}_{1}(t)$. Thus, we have

$$
\int_{t_{0}}^{t_{2}} A_{n}\left(q_{1}, \dot{q}_{1}\right) d t \geq \int_{t_{0}}^{t_{2}} A_{n}\left(\bar{q}_{1}, \dot{\bar{q}}_{1}\right) d t
$$

We compare the action $\int_{t_{0}}^{t_{2}} A_{n}\left(\tilde{q}_{1}, \dot{\tilde{q}}_{1}\right) d t$ with the action $\int_{t_{0}}^{t_{2}} A_{n}\left(\bar{q}_{1}, \dot{\bar{q}}_{1}\right) d t$ in the alternative cases, which is based on the choices of $\tilde{t}_{1}$. See Fig.3, where $\bar{t}=\frac{t_{0}+t_{2}}{2}$.

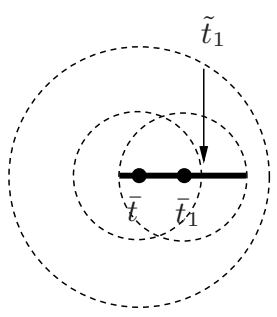

Case 1

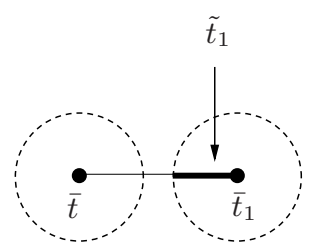

Case 2a

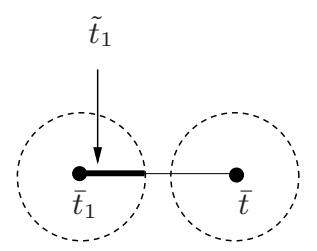

Case 2b

Figure 3: The choices of $\tilde{t}_{1}$

Case 1: $\left|\bar{t}_{1}-\bar{t}\right| \leq \frac{C_{0}}{n}$ 
In this case, the average speed of $\bar{q}_{1}$ on $\left(t_{0}, \bar{t}_{1}\right)$ and $\left(\bar{t}_{1}, t_{2}\right)$ have the same quantity order as $\left|\omega_{1}\right|$. By $\left|\tilde{t}_{1}-\bar{t}_{1}\right| \leq \frac{C_{0}}{n}$, we have $\left|\tilde{t}_{1}-\bar{t}\right| \leq 2 \frac{C_{0}}{n}$. Hence the average speed of $\tilde{q}_{1}$ on $\left(t_{0}, \tilde{t}_{1}\right)$ and $\left(\tilde{t}_{1}, t_{2}\right)$ have also the same quantity order as $\left|\omega_{1}\right|$. Thus, Lemma 2.4 implies

$$
\int_{t_{0}}^{t_{2}} A_{n}\left(\tilde{q}_{1}, \dot{\tilde{q}}_{1}\right) d t-\int_{t_{0}}^{t_{2}} A_{n}\left(\bar{q}_{1}, \dot{\bar{q}}_{1}\right) d t \leq \frac{C_{5}}{n} \sigma_{n} \exp \left(-\frac{C_{6} \sqrt{\sigma_{n}}}{\left|\omega_{1}\right|}\right) .
$$

Case 2: $\left|\bar{t}_{1}-\bar{t}\right|>\frac{C_{0}}{n}$.

In this case, we take $\tilde{t}_{1}$ such that $\left|\tilde{t}_{1}-\bar{t}\right| \leq\left|\bar{t}_{1}-\bar{t}\right|$, which can be achieved by the suitable choice of the position of $\tilde{q}_{1}\left(\tilde{t}_{1}\right)$. More precisely,

- if $\bar{t}_{1}>\bar{t}+\frac{C_{0}}{n}$ (Case 2a in Fig.2), we choose $\tilde{t}_{1}$ as the last time before $\bar{t}_{1}$, corresponding to $\left(\tilde{q}_{1}\left(\tilde{t}_{1}\right), q_{2}\left(\tilde{t}_{1}\right)\right)=\tilde{x}_{1}^{\prime}$ in Fig.2;

- if $\bar{t}_{1}<\bar{t}-\frac{C_{0}}{n}$ (Case 2b in Fig.2), we choose $\tilde{t}_{1}$ as the first time after $\bar{t}_{1}$, corresponding to $\left(\tilde{q}_{1}\left(\tilde{t}_{1}\right), q_{2}\left(\tilde{t}_{1}\right)\right)=\tilde{x}_{1}^{\prime \prime}$ in Fig.2.

For Case 2a, $\tilde{t}_{1} \in\left[\bar{t}, \bar{t}_{1}\right]$. For Case $2 \mathrm{~b}, \tilde{t}_{1} \in\left[\bar{t}_{1}, \bar{t}\right]$. From Remark 2.5, it follows that $L\left(\tilde{t}_{1}\right)-L(\bar{t}) \leq 0$, i.e.

$$
\int_{t_{0}}^{t_{2}} A_{n}\left(\tilde{q}_{1}, \dot{\tilde{q}}_{1}\right) d t-\int_{t_{0}}^{t_{2}} A_{n}\left(\bar{q}_{1}, \dot{\bar{q}}_{1}\right) d t \leq 0
$$

Hence, for any $\bar{t}_{1} \in\left(t_{0}, t_{2}\right)$, we can find $\tilde{t}_{1}$ such that

$$
\begin{aligned}
\int_{t_{0}}^{t_{2}} A_{n}\left(\tilde{q}_{1}, \dot{\tilde{q}}_{1}\right) d t-\int_{t_{0}}^{t_{2}} A_{n}\left(q_{1}, \dot{q}_{1}\right) d t & \leq \int_{t_{0}}^{t_{2}} A_{n}\left(\tilde{q}_{1}, \dot{\tilde{q}}_{1}\right) d t-\int_{t_{0}}^{t_{2}} A_{n}\left(\bar{q}_{1}, \dot{\bar{q}}_{1}\right) d t \\
& \leq \frac{C_{5}}{n} \sigma_{n} \exp \left(-\frac{C_{6} \sqrt{\sigma_{n}}}{\left|\omega_{1}\right|}\right)
\end{aligned}
$$

Since

$$
\left|\omega_{1}\right| \leq n^{-\frac{a}{2}-\epsilon} .
$$

It is easy to see that for $n$ large enough,

$$
\frac{C_{5}}{n} \sigma_{n} \exp \left(-\frac{C_{6} \sqrt{\sigma_{n}}}{\left|\omega_{1}\right|}\right) \leq\left|\omega_{1}\right|^{\lambda}
$$

where $\sigma_{n}=n^{-a}$, which contradicts to (2.16) for large $n$. This completes the proof of Lemma 2.1 .

\section{Destruction of Lagrangian torus with an arbitrary rotation VECTOR}

By (1.1), for every non resonant rotation vector $\omega=\left(\omega_{1}, \ldots, \omega_{d}\right)(d \geq 2)$, there exists a sequence of integer vector $k_{n} \in \mathbb{Z}^{d}$ satisfying $\left|k_{n}\right| \rightarrow \infty$ as $n \rightarrow \infty$ such that

$$
\left|\left\langle k_{n}, \omega\right\rangle\right|<\frac{C}{\left|k_{n}\right|^{d-1}} .
$$


Lemma 3.1 There exists an integer vector $k_{n}^{\prime}$ such that

$$
\left\langle k_{n}, k_{n}^{\prime}\right\rangle=0, \quad\left|k_{n}^{\prime}\right| \sim\left|k_{n}\right| \text { and }\left|\left\langle k_{n}^{\prime}, \omega\right\rangle\right| \sim\left|k_{n}\right| .
$$

Proof For $d=2$, Lemma 3.1 holds obviously. In fact, it suffices to consider $k_{n}^{\prime} \in \mathbb{Z}^{3}$. Let $k_{n}^{\prime}=\left(k_{n 1}^{\prime}, k_{n 2}^{\prime}, k_{n 3}^{\prime}\right)$ be the integer vector satisfying (3.1), then for $k_{n}^{\prime} \in \mathbb{Z}^{d}$, one can take $k_{n}^{\prime}=\left(k_{n 1}^{\prime}, k_{n 2}^{\prime}, k_{n 3}^{\prime}, 0, \ldots, 0\right)$ to verify Lemma 3.1.

Since $\omega$ is non-resonant, then $\left|k_{n}\right| \rightarrow \infty$, for $n \rightarrow \infty$. Let $\Pi$ be the plane orthogonal with respect to $k_{n}$. Let $S_{R \alpha} \subset \Pi$ be the sector with central point $(0,0,0)$, central angle $\alpha$ and radius $R$ satisfying $\alpha \sim 1$. Let the angle between $\omega$ and one of the radii of $S_{R \alpha}$ be $\beta$, where $\beta \sim 1$ and $\beta \gg \alpha$. Without loss of generality, we assume $k_{n 1} \cdot k_{n 2} \cdot k_{n 3} \neq 0$. Let $e_{1}=\left(-k_{n 2}, k_{n 1}, 0\right), e_{2}=\left(0,-k_{n 3}, k_{n 2}\right)$, then $e_{1}, e_{2}$ are the generators of the hyperplane $\left\langle x, k_{n}\right\rangle=0$ and satisfy $\left|e_{1}\right| \leq\left|k_{n}\right|,\left|e_{2}\right| \leq\left|k_{n}\right|$. Hence, we take $R \sim\left|k_{n}\right|$, then it is easy to see that the sector $S_{R \alpha}$ contains at least one integer point $m=\left(m_{1}, m_{2}, m_{3}\right)$ satisfying $|m| \sim\left|k_{n}\right|$ for $n$ large enough.

We take $k_{n}^{\prime}=\left(m_{1}-0, m_{2}-0, m_{3}-0\right)$, then $\left|k_{n}^{\prime}\right| \sim\left|k_{n}\right|$. Let $\theta$ be the angle between $k_{n}^{\prime}$ and $\omega$, then $\theta \sim 1$. Hence,

$$
\left|\left\langle k_{n}^{\prime}, \omega\right\rangle\right|=\left|k_{n}^{\prime}\right||\omega||\cos \theta| \sim\left|k_{n}\right|
$$

which together with $\left\langle k_{n}, k_{n}^{\prime}\right\rangle=0$ and $\left|k_{n}^{\prime}\right| \sim\left|k_{n}\right|$ implies that the integer satisfying (3.1) does exist.

\subsection{Transformation of coordinates}

By Lemma 3.1, we choose a sequence of $k_{n} \in \mathbb{Z}^{d}$ satisfying (1.1) and an integer vector sequence $k_{n}^{\prime}$ such that $\left\langle k_{n}^{\prime}, k_{n}\right\rangle=0,\left|k_{n}^{\prime}\right| \sim\left|k_{n}\right|$ and $\left|\left\langle k_{n}^{\prime}, \omega\right\rangle\right| \sim\left|k_{n}\right|$. In addition, select $d-2$ integer vectors $l_{n 3}, \ldots, l_{n d}$ such that $k_{n}, k_{n}^{\prime}, l_{n 3}, \ldots, l_{n d}$ are pairwise orthogonal. Let

$$
K_{n}=\left(k_{n}, k_{n}^{\prime}, l_{n 3}, \ldots, l_{n d}\right)^{t} .
$$

We choose the transformation of the coordinates

$$
q=K_{n} x
$$

Let $p$ denotes the dual coordinate of $q$ in the sense of Legendre transformation, i.e. $p=\frac{\partial L}{\partial \dot{q}}$, it follows that

$$
y=K_{n}^{t} p,
$$

where $K_{n}^{t}$ denotes the transpose of $K_{n}$. We set

$$
\Phi_{n}=\left(\begin{array}{cc}
K_{n} & \\
& K_{n}^{-t}
\end{array}\right),
$$

then

$$
\left(\begin{array}{l}
q \\
p
\end{array}\right)=\Phi_{n}\left(\begin{array}{l}
x \\
y
\end{array}\right) .
$$

It is easy to verify that

$$
\Phi_{n}^{t} J_{0} \Phi_{n}=J_{0},
$$


where

$$
J_{0}=\left(\begin{array}{cc}
\mathbf{0} & \mathbf{1} \\
-\mathbf{1} & \mathbf{0}
\end{array}\right)
$$

where 1 denotes a $d \times d$ unit matrix. Hence, $\Phi_{n}$ is a symplectic transformation in the phase space.

Lemma 3.2 If the Hamiltonian flow generated by $\tilde{H}_{n}(x, y)$ admits a Lagrangian torus with rotation vector $\omega$, then the Hamiltonian flow generated by $H_{n}(q, p)$ also admits a Lagrangian torus with rotation vector $K_{n} \omega$, where $(q, p)^{t}=\Phi_{n}(x, y)^{t}$.

Proof Let $\tilde{\mathcal{T}}^{d}$ be the Lagrangian torus admitted by $\tilde{H}_{n}(x, y)$, a symplectic form $\Omega$ vanishes on $\mathrm{T}_{x} \tilde{\mathcal{T}}^{d}$ for every $x \in \tilde{\mathcal{T}}^{d}$. Since $K_{n}$ consists of integer vectors, then $\mathcal{T}^{d}:=K_{n} \tilde{\mathcal{T}}^{d}$ is still a torus. $\Phi_{n}$ is a symplectic transformation, hence $\mathcal{T}^{d}$ is a Lagrangian torus. From Definition 1.3. each orbit on $\tilde{\mathcal{T}}^{d}$ has the same rotation vector $\omega$. Let $\tilde{\gamma}(t)$ be a lift of an orbit on $\tilde{\mathcal{T}}^{d}$, it follows that

$$
\omega=\lim _{t \rightarrow \infty} \frac{\tilde{\gamma}(t)-\tilde{\gamma}(-t)}{2 t} .
$$

For $\gamma(t)=K_{n} \tilde{\gamma}(t)$, we have

$$
\begin{aligned}
\lim _{t \rightarrow \infty} \frac{\gamma(t)-\gamma(-t)}{2 t} & =\lim _{t \rightarrow \infty} \frac{K_{n} \tilde{\gamma}(t)-K_{n} \tilde{\gamma}(-t)}{2 t} ; \\
& =K_{n} \lim _{t \rightarrow \infty} \frac{\tilde{\gamma}(t)-\tilde{\gamma}(-t)}{2 t} \\
& =K_{n} \omega .
\end{aligned}
$$

This completes the proof.

\subsection{Proof of Theorem 1.1}

We construct $\tilde{H}_{n}(x, y)$ as follow:

$$
\tilde{H}_{n}(x, y)=\frac{1}{2}|y|^{2}-\tilde{P}_{n}(x),
$$

where

$$
\tilde{P}_{n}(x)=\frac{1}{\left|k_{n}\right|^{a+2}}\left(1-\cos \left\langle k_{n}, x\right\rangle\right)+\frac{1}{\left|k_{n}\right|^{2}} v_{n}\left(\left\langle k_{n}, x\right\rangle,\left\langle k_{n}^{\prime}, x\right\rangle\right),
$$

where $k_{n}^{\prime}$ is the second row of $K_{n}$ and $v_{n}$ is defined by (2.3). Let $q=K_{n} x$. In particular, we have

$$
\left\{\begin{array}{l}
q_{1}=\left\langle k_{n}, x\right\rangle, \\
q_{2}=\left\langle k_{n}^{\prime}, x\right\rangle .
\end{array}\right.
$$

By the transformation of coordinates and the Legendre transformation, the Lagrangian function corresponding to (3.3) is

$$
\begin{aligned}
L_{n}\left(q_{1}, Q, \dot{q}_{1}, \dot{Q}\right)= & \frac{1}{2} \sum_{i=3}^{d} \frac{\left|\dot{q}_{i}\right|^{2}}{\left|l_{n i}\right|^{2}}+\frac{\left|\dot{q}_{2}\right|^{2}}{2\left|k_{n}^{\prime}\right|^{2}} \\
& +\frac{1}{\left|k_{n}\right|^{2}}\left(\frac{1}{2}\left|\dot{q}_{1}\right|^{2}+\frac{1}{\left|k_{n}\right|^{a}}\left(1-\cos \left(q_{1}\right)\right)+v_{n}\left(q_{1}, q_{2}\right)\right),
\end{aligned}
$$


where $Q=\left(q_{2}, \ldots, q_{d}\right)$.

For the Hamiltonian flow generated by (3.3), by Lemma 3.2, for the destruction of the Lagrangian torus $\tilde{\mathcal{T}}^{d}$ with rotation vector $\omega$, it is sufficient to prove that the Euler-Lagrange flow generated by (3.5) admits no the Lagrangian torus $\mathcal{T}^{d}:=K_{n} \tilde{\mathcal{T}}^{d}$ with rotation vector $K_{n} \omega$. Let $K_{n} \omega=\left(\omega_{1}, \ldots, \omega_{d}\right)$.

Replacing $n$ by $\left|k_{n}\right|$ in the proof of Lemma 2.1, we have that the Euler-Lagrange flow generated by (3.5) does not admit any Lagrangian torus with rotation vector satisfying

$$
\left|\omega_{1}\right|<\left|k_{n}\right|^{-\frac{a}{2}-\epsilon} \text {. }
$$

Here, it should be noted that since $\left|k_{n}^{\prime}\right| \sim\left|k_{n}\right|$ and the argument in Section 2 only involves first two components of the system, then it still holds for (3.5). From the construction of $K_{n},\left|\omega_{1}\right|=\left|\left\langle k_{n}, \omega\right\rangle\right|$ which together with (1.1) implies

$$
\left|\omega_{1}\right|<\frac{C}{\left|k_{n}\right|^{d-1}} .
$$

Based on Lemma 2.1, it suffices to take

$$
\frac{C}{\left|k_{n}\right|^{d-1}} \leq\left|k_{n}\right|^{-\frac{a}{2}-\epsilon}
$$

which implies

$$
a<2 d-2-2 \epsilon .
$$

It follows from (2.3) and (3.3) that

$$
\begin{aligned}
& \left\|\tilde{H}_{n}(x, y)-H_{0}(y)\right\|_{C^{r}} \\
& \quad=|| \tilde{P}_{n}(x) \|_{C^{r}} \\
& \quad=\left|k_{n}\right|^{-2}\left(\left|k_{n}\right|^{-a}|| 1-\cos \left\langle k_{n}, x\right\rangle\left\|_{C^{r}}+\right\| v_{n}\left(\left\langle k_{n}, x\right\rangle,\left\langle k_{n}^{\prime}, x\right\rangle\right) \|_{C^{r}}\right), \\
& \quad \leq\left|k_{n}\right|^{-2}\left(C_{1}\left|k_{n}\right|^{-a+r}+C_{2}\left|k_{n}\right|^{-s^{\prime}(d-1)+r}\right), \\
& \quad \leq C_{3}\left(\left|k_{n}\right|^{r-a-2}+\left|k_{n}\right|^{r-3(d-1)-2}\right),
\end{aligned}
$$

where $\sigma$ is a small positive constant independent of $n$. To complete the proof of Theorem 1.1, it is enough to make $r-a-2<0$ and $r-3(d-1)-2<0$, which together with (3.6) implies

$$
r<2 d-2 \epsilon .
$$

Taking $\delta=3 \epsilon$, this completes the proof of Theorem 1.1.

Remark 3.3 Based on the strategy of the proof, it is easy to see that the completely integrable part $\frac{1}{2}|y|^{2}$ of (3.3) can be generalized to $\frac{1}{2}\langle M y, y\rangle$, where $M=$ $\operatorname{diag}\left(m_{1}, \ldots, m_{d}\right)$ and $m_{i}(i=1, \ldots, d)$ are positive constants independent of $n$. If $M$ is a general positive definite matrix, some technical difficulties would appear.

Acknowledgement The authors sincerely thank the referees for their careful reading of the manuscript and invaluable comments which were very helpful in improving this paper. The authors also would like to thank Ugo Bessi for illustrations to his results $[\mathrm{Be}$. This work is under the support of the NNSF of China (Grant 11171146), Basic Research Programme of Jiangsu Province, China (BK2008013), PAPD of Jiangsu Province of China and Research and Innovation Project for College Graduates of Jiangsu Province (CXZZ12_0030). 


\section{REFERENCES}

[Be] U.Bessi. An analytic counterexample to KAM theorem. Ergod. Th. \& Dynam. Sys. 20 (2000), 317-333.

[BK] D.Bernstein and A.Katok. Birkhoff periodic orbits for small perturbation of completely integrable Hamiltonian systems with convex Hamiltonians. Invent. Math. 88 (1987), 225-241.

[BP] M.Bialy and L.Polterovich. Hamiltonian systems, Lagrangian tori and Birkhoff's theorem. Math. Ann. 292 (1992), 619-627.

[C] C.-Q.Cheng. Non-existence of KAM torus. Acta Mathmatica Sinica. 27 (2011), 397-404.

[F] G.Forni. Analytic destruction of invariant circles. Ergod. Th. \& Dynam. Sys. 14 (1994), 267-298.

[H1] M.R.Herman. Sur les courbes invariantes par les difféomorphismes de l'anneau. Astérisque 103-104 (1983), 1-221.

[H2] M.R.Herman. Sur les courbes invariantes par les difféomorphismes de l'anneau. Astérisque 144 (1986), 1-243.

[H3] M.R.Herman. Non existence of Lagrangian graphs. unpublished preprint (1990).

[H4] M.R.Herman. Inégalités "a priori" pour des tores lagrangiens invariants par des difféomrphismes symplectiques. Inst. Hautes Études Sci. Publ. Math. 70 (1990), 47-101.

[Ma1] J.N.Mather. Destruction of invariant circles. Ergod. Th. \& Dynam. Sys. 8 (1988), 199-214.

[Mo1] J.Moser. On invariant curves of area-preserving mappings of an annulus. Nachr. Akad. Wiss. Göttingen, II. Math-Phys. KL. 1 (1962), 1-20.

[Mo2] J.Moser. A rapidly convergent iteration method and non-linear partail differential equations I, II. Annali della Scuola Normale Superiore di Pisa. 20 (1966), 265315.

[Mo3] J.Moser. On the construction of almost periodic solutions for ordinary differential equations. Proceedings of the International Conference on Functional Analysis and Related Topics, Tokyo. (1969), 60-67.

[P] J.Pöschel. Integrability of Hamiltonian systems on Cantor sets. Comm. Pure Appl. Math. 35 (1982), 653-696.

[R1] H.Rüssman. On optimal estimates for the solutions of linear partial differential equations of first order with constant coefficients on the torus. Lecture Notes in Physics. 38 (1975), 598-624.

[R2] H.Rüssman. On the existence of invariant curves of twist mappings of an annulus. Lecture Notes in Mathematics. 1007 (1983), 677-718.

[W] L.Wang. Variational destruction of invariant circles. Discrete and Continuous Dynamical Systems-A. 32 (2012), 4429-4443.

Department of Mathematics, Nanjing University, Nanjing 210093, CHINA.

E-mail address: chengcq@nju.edu.cn CHINA.

Department of Mathematics, Nanjing University, Nanjing 210093,

E-mail address: linwang.math@gmail.com 